\title{
Increasing power-law range in avalanche amplitude and energy distributions
}

\author{
Víctor Navas-Portella, ${ }^{1,2,3}$ Isabel Serra, ${ }^{1}$ Álvaro Corral,,${ }^{1,2,4,5}$ and Eduard Vives ${ }^{6,7}$ \\ ${ }^{1}$ Centre de Recerca Matemàtica, Edifici C, Campus Bellaterra, E-08193 Bellaterra, Catalonia, Spain \\ ${ }^{2}$ Barcelona Graduate School of Mathematics, Edifici C, Campus Bellaterra, E-08193 Barcelona, Spain \\ ${ }^{3}$ Facultat de Matemàtiques i Informàtica, Universitat de Barcelona, Gran Via de les Corts Catalanes, 585, E-08007 Barcelona, Spain \\ ${ }^{4}$ Complexity Science Hub Vienna, Josefstädter Straße 39, 1080 Vienna, Austria \\ ${ }^{5}$ Departament de Matemàtiques, Universitat Autònoma de Barcelona, E-08193 Barcelona, Spain \\ ${ }^{6}$ Departament de Matèria Condensada, Facultat de Física, Universitat de Barcelona, Martí Franquès 1, 08028 Barcelona, Catalonia, Spain \\ ${ }^{7}$ Universitat de Barcelona Institute of Complex Systems, Facultat de Física, Universitat de Barcelona, Barcelona, Catalonia, Spain
}

(Received 20 November 2017; published 21 February 2018)

\begin{abstract}
Power-law-type probability density functions spanning several orders of magnitude are found for different avalanche properties. We propose a methodology to overcome empirical constraints that limit the range of truncated power-law distributions. By considering catalogs of events that cover different observation windows, the maximum likelihood estimation of a global power-law exponent is computed. This methodology is applied to amplitude and energy distributions of acoustic emission avalanches in failure-under-compression experiments of a nanoporous silica glass, finding in some cases global exponents in an unprecedented broad range: 4.5 decades for amplitudes and 9.5 decades for energies. In the latter case, however, strict statistical analysis suggests experimental limitations might alter the power-law behavior.
\end{abstract}

DOI: 10.1103/PhysRevE.97.022134

\section{INTRODUCTION}

Avalanche processes are present in a vast number of out-ofequilibrium physical phenomena [1-3]. These processes are characterized by intense bursts of activity preceded by periods of silence and can be described in terms of probability density functions (PDFs) that exhibit lack of finite moments due to their power-law shape. Consequently, fitting PDFs to different avalanche properties such as size, energy, or amplitude is a task that requires a rigorous treatment $[4,5]$. One of the most important features of this kind of functions is their invariance under any scale transformation which can be written as $f(\lambda x)=\lambda^{-\gamma} f(x)$ for $x \in(0,+\infty)$. The only solution for all $\lambda$ of this functional equation [6] is a power-law $f(x)=C x^{-\gamma}$, where the exponent $\gamma$ can take any real value and $C$ is a constant.

Some experimental works claim the presence of scale invariance with power-law behavior which scarcely covers a few orders of magnitude [7-9]. However, the shorter the powerlaw range the less reliable the property of scale invariance $[10,11]$. The distributions of amplitude of acoustic emission (AE) avalanches have been studied in different experimental works [12-16]. Nevertheless, experimental fitted distributions expand by at most two orders of magnitude in voltages $[12,16-18]$ due to the limitations in the observation windows. Typically the existence of noise and/or undercounting effects affects the smallest observable values, whereas saturation and/or lack of statistics due to undersampling limits the largest observable values. In most cases, these experimental limitations introduce cutoffs in the distributions that usually are not sharp due, for instance, to electronic uncertainties. Recent studies regarding the $\mathrm{AE}$ in compression experiments of porous materials [19-21], wood [22], ethanol-dampened charcoal [23], confined-granular matter under continuous shear [24], etc., have focused the attention in the energy distribution of avalanches due to the similarities with the Gutenberg-Richter law for earthquakes [25].

In this paper, we devise a procedure to broaden the range of validity of power-law-like behavior of the distributions corresponding to avalanche amplitudes and energies. From a set of $n_{\text {cat }}$ catalogs of events the measured properties of which span different observation windows, data analysis is performed by assembling them in order to obtain global exponents that characterize the distribution of these avalanche properties. Through this procedure, the fitted global distribution spans a broader range than the ones from the fit of every individual catalog.

This paper is organized as follows: In Sec. II an overview of the fitting procedure is shown. In Sec. III we present the experimental methodology in the recording of $\mathrm{AE}$ during displacement-driven compression of porous glasses [21]. In Secs. IV and V avalanche amplitudes and energies are studied, respectively, by applying the methodology given in Sec. II. Finally, a brief summary of the results is presented in Sec. VI.

\section{GENERAL METHODOLOGY}

By considering $n_{\text {cat }}$ catalogs of $N_{i}\left(i=1, \ldots, n_{\text {cat }}\right)$ events each, corresponding to different experiments (or different observation windows) and characterized by a set of properties (amplitude, energy, duration, etc.), one wants to fit a general power-law-type PDF with a global exponent for all the catalogs. Note that, in the $i$ th catalog, the variable $\mathcal{X}$ can acquire values in a range typically spanning several orders of magnitude. The first step consists in fitting a power-law PDF in a range $\left[a_{i}, b_{i}\right]$ for each catalog via maximum likelihood estimation (MLE) and goodness-of-fit testing [5]. Details of 
this fitting procedure are explained in Appendix A. By this method we correct for problems close to the limits of the observation windows discarding some experimental data.

Let us justify the use of truncated power-law distributions for the variables of interest:

$$
f_{\mathcal{X}}^{(i)}(x) d x=\frac{1-\gamma_{i}}{b_{i}^{1-\gamma_{i}}-a_{i}^{1-\gamma_{i}}} x^{-\gamma_{i}} d x \text {, where } x \in\left[a_{i}, b_{i}\right] .
$$

By truncated we mean that the power law is truncated from below and from above, i.e., the power law only holds for the range $a_{i} \leqslant x \leqslant b_{i}$. Standard (usual) power laws are only truncated from below (due to normalization [4]); however, in many real systems considerable deviations from power-law behavior appear in the tail of the distributions. Deviations from criticality can be simply modeled with an exponential tail, as $f_{\mathcal{X}}^{(i)}(x) \propto$ $e^{-x / c_{i}} / x^{\gamma_{i}}$ [27]; nevertheless, other factors, such as finite-size effects [28], or such as saturation (or the combination of them), may require more sophisticated modeling. A way to avoid the difficulties in the parametrization of the tail is provided by (doubly) truncated power laws [5], with an upper cutoff $b_{i}$ well below the crossover point to the non-power-law tail $c_{i}$.

Once the particular truncated power-law fits have been done, one may be able to state that, for the $i$ th catalog, the variable $\mathcal{X}$ follows a power-law $\operatorname{PDF} f_{\mathcal{X}}^{(i)}\left(x ; \hat{\gamma}_{i}, a_{i}, b_{i}\right)$ in a certain range $\left[a_{i}, b_{i}\right]$ with exponent $\hat{\gamma}_{i}$ and a number $\hat{n}_{i}$ of data entering into the fit, with $\hat{n}_{i} \leqslant N_{i}$ (for convenience, in the notation we stress the dependence of the PDF on the parameters). The next step is to formulate the null hypothesis $H_{0}$ : the variable $\mathcal{X}$ is powerlaw distributed with a global exponent $\Gamma$ for all the catalogs.

The log-likelihood function of this global distribution can be written as

$$
\log \mathcal{L}=\sum_{i=1}^{n_{\text {cat }}} \sum_{j=1}^{\hat{n}_{i}} \log f_{\mathcal{X}}^{(i)}\left(x_{i j} ; \Gamma, a_{i}, b_{i}\right)
$$

where $x_{i j}$ corresponds to the values of the variable $\mathcal{X}$ in the $i$ th catalog, $\hat{n}_{i}$ is the number of data between $a_{i}$ and $b_{i}$ in the $i$ th catalog, and $\Gamma$ is the global exponent. Since the particular ranges $\left[a_{i}, b_{i}\right]$ and the number of data $\hat{n}_{i}$ are known, one has to find the value of the exponent $\hat{\Gamma}$ that maximizes the loglikelihood expression in Eq. (2).

Intuitively, one could be tempted to think that the null hypothesis will not be rejected if the values of the particular exponents $\gamma_{i}$ do not differ too much. Nevertheless, a more rigorous treatment is required. Statistical procedures, such as a permutational test [29], could be used in order to check whether the exponents are the same or not. However, since we propose a global distribution characterized by a global exponent $\hat{\Gamma}$, a goodness-of-fit test for this global distribution is performed in order to determine whether the null hypothesis can be rejected or not $[4,5]$. If the goodness-of-fit test yields a high enough $p$ value, one is able to state that the variable $\mathcal{X}$ is power-law distributed with exponent $\hat{\Gamma}$ along all the different catalogs or experiments, with ranges $\left[a_{i}, b_{i}\right]$ each. Details of the global goodness-of-fit test are given in Appendix B. In this way, if these intervals span different orders of magnitude, one can increase the power-law range by several decades. An alternative procedure, where the ranges $\left[a_{i}, b_{i}\right]$ are optimized directly from Eq. (2), is disregarded for being enormously computer-time consuming.
This methodology is applied to avalanche amplitudes and energies on AE data in failure-under-compression experiments of nanoporous silica glasses. Since the experimental setup records discrete values for the amplitude (in $\mathrm{dB}$ ) and almost continuous values of the energy (in aJ), particular expressions for the log likelihood Eq. (2) as well as the different ways of implementing the goodness-of-fit test will be explained in Secs. IV and V.

\section{FAILURE UNDER COMPRESSION OF POROUS GLASSES}

Uniaxial compression experiments of porous glass Vycor (a nanoporous silica glass with $40 \%$ porosity) are performed in a conventional test machine ZMART.PRO (Zwick/Roell). Cylindrical samples with no lateral confinement are placed between two plates that approach each other at a certain constant rate $\dot{z}$. We refer to such a framework as displacement-driven compression. With the aim of having the same conditions for all the experiments, samples have the same diameters $\Phi=4.45 \mathrm{~mm}$ and heights $H=8 \mathrm{~mm}$, and the compression rate is fixed at $\dot{z}=0.005 \mathrm{~mm} / \mathrm{min}$. Before compression, samples were cleaned following the standard procedure: immersed in a $30 \%$ solution of $\mathrm{H}_{2} \mathrm{O}_{2}$ over the course of $24 \mathrm{~h}$ and dried with a 8 -h temperature ramp from room temperature to $130^{\circ} \mathrm{C}$. This protocol ensures that thirsty porous glass samples are clean of humidity and other organic impurities. Simultaneous to the compression, recording of an AE signal is performed by using a piezoelectric transducer embedded in one of the compression plates. The electric signal $U(t)$ is preamplified, band filtered (between $20 \mathrm{kHz}$ and $2 \mathrm{MHz}$ ), and analyzed by means of a PCI-2 acquisition system from Euro Physical Acoustics (Mistras Group) with an analog-to-digital (AD) card working at 40 megasamples per second with 18 bits precision [30]. This should be kept in mind when considering some of the measures as continuous (energy or voltage). Recording of data stops when a big failure event occurs and the sample gets destroyed.

We prescribe that an $\mathrm{AE}$ avalanche event (often called an $\mathrm{AE}$ hit in specialized $\mathrm{AE}$ literature) starts at the time $t_{j}$ when the signal $U(t)$ crosses a fixed detection threshold and finishes at time $t_{j}+\tau_{j}$ when the signal remains below threshold from $t_{j}+$ $\tau_{j}$ to at least $t_{j}+\tau_{j}+200 \mu \mathrm{s}$. The amplitude $A$ recorded in $\mathrm{dB}$ follows the expression $A=\left[20 \log _{10}\left(|V| / V_{0}\right)\right]$, where $V$ is the peak voltage achieved by the AE signal during the event, $V_{0}=$ $1 \mu \mathrm{V}$ is a reference voltage, and the brackets round the value to its nearest integer in $\mathrm{dB}$. Such a procedure is extensively used in electronic systems. Note that in our terminology $A$ will be called amplitude in $d B$, whereas the peak voltage $V$ will be referred to simply as amplitude, in agreement with the literature. From the values of $A$ one can obtain the values $y$ of the discretized peak voltage:

$$
y=g(A)=V_{0} \times 10^{A / 20} .
$$

As the values of $A$ are integer, the values of $y$ will no longer be integer but they will collapse into a set of values $\left\{y_{1}, y_{2}, \ldots, y_{k}\right\}$ measured in $\mu \mathrm{V}$. The energy $E_{j}$ of each avalanche or event is determined as $E_{j}=\frac{1}{R} \int_{t_{j}}^{t_{j}+\tau_{j}} U^{2}(t) d t$ where $R$ is a reference resistance of $10 \mathrm{k} \Omega$. At the end of one experiment, one has a catalog or collection of events each of them characterized 


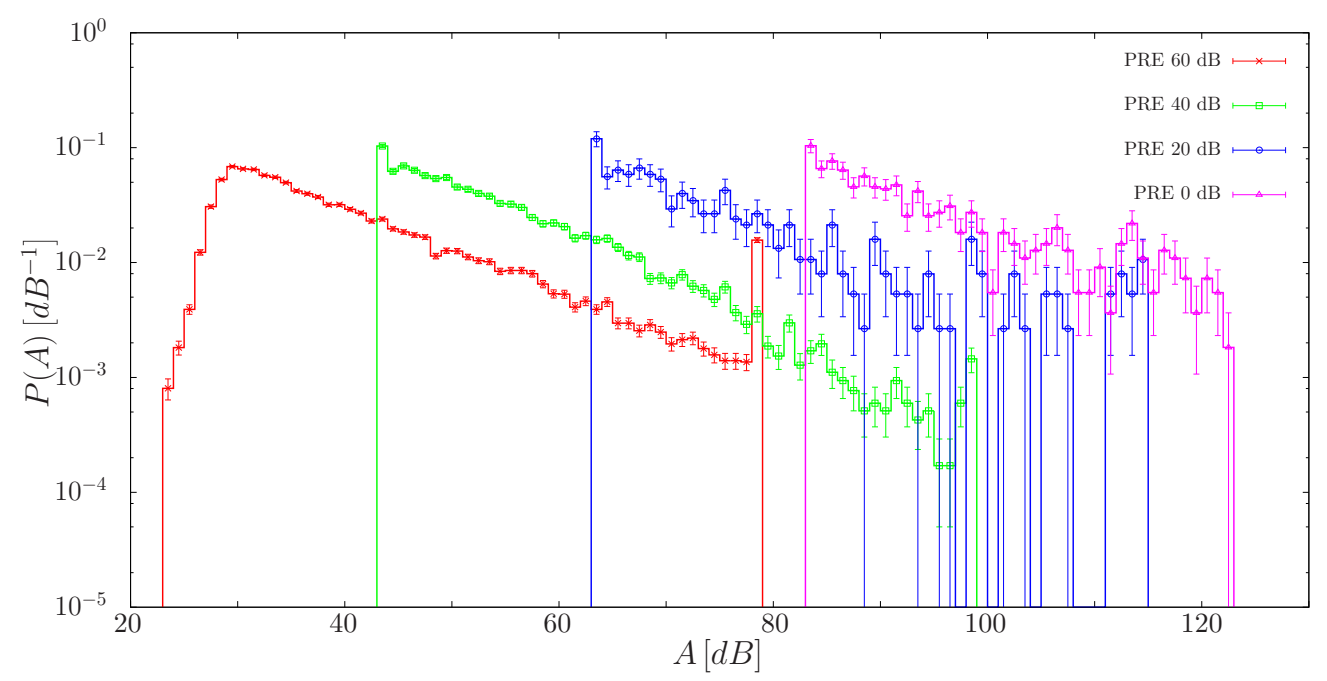

FIG. 1. Estimated probability mass functions of the amplitude in $\mathrm{dB}$ for the complete datasets of the different experiments performed at different preamplifications (PRE). Error bars correspond to one standard deviation [5].

by a time of occurrence $t$, amplitude in $\mathrm{dB} A$, energy $E$, and duration $\tau$.

In order to obtain catalogs that span different observation windows, displacement-driven compression experiments with Vycor cylinders have been performed for different values of the preamplification and the detection threshold. In this case, $n_{\text {cat }}=4$ experiments have been performed with the following preamplification values: $60,40,20$, and $0 \mathrm{~dB}$ and the respective values of the detection threshold $23,43,63$, and $83 \mathrm{~dB}$ referring to the signal $U(t)$, not the preamplified signal (in such a way that after preamplification the threshold always moves to 83 $\mathrm{dB})$. These are values of the threshold that are as low as possible in order to avoid external noise, mostly of electronic origin in the AD card.

Signal preamplification is necessary if one wants to record small AE events. Some values of the preamplified signals turn so large that they cannot be detected correctly by the acquisition system. This fact leads to a saturation in the amplitude and, consequently, an underestimated energy of the AE event. This effect can be immediately observed in the distributions of the amplitude in $\mathrm{dB}$, Fig. 1, where there is an excess of AE events in the last bin of amplitudes for the experiments at 60 and 40 $\mathrm{dB}$. Note that the thresholding we perform turns out to be of the same kind as that in Refs. [31-34].

\section{AMPLITUDES}

\section{A. Particular fits}

We consider that, for each experiment, the random variable $\mathcal{V}$ corresponding to amplitude (i.e., the peak voltage, the values of which are denoted by $V$ ) follows a truncated continuous power-law distribution:

$f_{\mathcal{V}}(V) d V=\frac{1-\alpha}{V_{\max }^{1-\alpha}-V_{\min }^{1-\alpha}} V^{-\alpha} d V$, where $V \in\left[V_{\min }, V_{\max }\right]$.

However, the true value of $\mathcal{V}$ is not accessible from the experiments, and what we have instead is its discretized counterpart $\mathcal{Y}$ (the discretized peak voltage, the values of which are denoted by $y$ ), which is concentrated in $k$ discrete values (but not equispaced). In fact, the values $V$ that the variable $\mathcal{V}$ can take are the real values of the voltages read by the AD card, but they are transformed into $\mathrm{dB}$, losing precision.

Under the assumption of a power-law distributed $\mathcal{V}$, we are able to state that the variable $\mathcal{Y}$ has probability mass function

$$
\begin{aligned}
f_{\mathcal{Y}}(y) & =P(\mathcal{Y}=y) \\
& =P[g(A-\Delta) \leqslant \mathcal{V}<g(A+\Delta)] \\
& =F_{\mathcal{V}}[g(A+\Delta)]-F_{\mathcal{V}}[g(A-\Delta)] \\
& =\frac{g^{1-\alpha}(A+\Delta)-g^{1-\alpha}(A-\Delta)}{V_{\max }^{1-\alpha}-V_{\min }^{1-\alpha}} \\
& =\frac{2 \sinh [2.30(1-\alpha) \Delta / 20]}{V_{\max }^{1-\alpha}-V_{\min }^{1-\alpha}} \frac{1}{y^{\alpha-1}},
\end{aligned}
$$

where $P$ refers to a probability, $y=g(A), \Delta=0.5 \mathrm{~dB}$ is a vicinity around the values of $A, 2.30 \simeq \log 10$, and $F_{\mathcal{V}}$ is the cumulative distribution function obtained from the integration of Eq. (4). Note that $f_{\mathcal{Y}}(y)$ is a power law but with exponent $\alpha-1$ because the length of the intervals $V$ is integrated over increase linearly in $y$.

The log-likelihood function for a particular experiment can be written as

$$
\begin{aligned}
\log \mathcal{L} & =\sum_{l=A_{\min }}^{A_{\max }} \omega_{l} \log f_{\mathcal{Y}}[g(l)] \\
& =\sum_{l=A_{\min }}^{A_{\max }} \omega_{l} \log \left(\frac{2 \sinh [2.30(1-\alpha) \Delta / 20]}{V_{\max }^{1-\alpha}-V_{\min }^{1-\alpha}} \frac{1}{y_{l}^{\alpha-1}}\right)
\end{aligned}
$$

where $y_{l}=g(l), A_{\min }$, and $A_{\max }$ are the values of the amplitude in $\mathrm{dB}$ corresponding to the cutoffs imposed on the sample for the analysis (see Appendix A for further details). The frequency $\omega_{l}$ is the number of events with discretized peak voltage $y_{l}=g(l)$. The next step consists in finding the value of $\alpha$ that maximizes Eq. (6) using a numerical method. The values of the fitted exponent $\alpha$ for different values of $A_{\min }$ and $A_{\max }$ are 
TABLE I. Fitted parameters for Eq. (4) for each particular experiment and for the global fit. $\hat{\alpha}$ corresponds to the fitted exponent in the range $\left[A_{\min }, A_{\max }\right]$ for which the goodness-of-fit test exceeds the significance level $p_{c}=0.2$. Numbers in parentheses correspond to the minimum and maximum value of the amplitude in $\mathrm{dB}$ for each sample. $\hat{n}$ is the number of data entering into the fit and $N$ is the total number of events in the dataset. The error of the exponent is computed as the standard deviation of the MLE [5].

\begin{tabular}{|c|c|c|c|c|c|}
\hline $\operatorname{PRE}(\mathrm{dB})$ & $\hat{\alpha}$ & $A_{\min }(\mathrm{dB})$ & $A_{\max }(\mathrm{dB})$ & $\hat{n}(N)$ & $p$ \\
\hline 60 & $1.743 \pm 0.007$ & $32(23)$ & 78 (79) & $21414(28614)$ & 0.92 \\
\hline 40 & $1.75 \pm 0.01$ & $46(43)$ & 72 (99) & 9146 (11717) & 0.20 \\
\hline 20 & $1.67 \pm 0.04$ & $64(63)$ & $114(115)$ & 353 (376) & 0.50 \\
\hline 0 & $1.61 \pm 0.04$ & $84(83)$ & 122 (123) & $528(548)$ & 0.64 \\
\hline Global & $1.740 \pm 0.006$ & $32(23)$ & $122(123)$ & 31441 (41255) & 0.36 \\
\hline
\end{tabular}

shown in Appendix C by using MLE exponent maps [35]. Once the exponent is found, one has to determine whether the fit is appropriate to data or not. All the details concerning the fitting procedure and the statistical test are given in Appendix A.

In Table I we present the fitted parameters of each particular experiment: exponents $\hat{\alpha}$, ranges $\left[A_{\min }, A_{\max }\right]$, number of events $\hat{n}$ included in the fit, and an estimated $p$ value. Each experiment detects avalanches within 2.8 decades in amplitude but all the experiments together would yield a total range of 5.8 decades. This range is broader than other ranges of AE amplitudes [13-17,36,37]. It must be mentioned that performing these particular fits by simply assuming that the discrete variable $\mathcal{Y}$ directly follows a truncated continuous power law leads to the rejection of this hypothesis in the goodness-of-fit test.

According to the range of detection for the experiment performed at $0 \mathrm{~dB}$, one could be able to observe events up to $139 \mathrm{~dB}$. Nevertheless, the maximum in this sample corresponds to $123 \mathrm{~dB}$. Under the hypothesis that a power-law distribution with the same exponent $(\hat{\alpha}=1.61 \pm 0.04)$ can be extended for larger values of the amplitude, the probability $P(123<A<140 \mathrm{~dB})$ turns out to be $P=0.042$. For $\hat{n}_{i}=$ 528 trials, the probability of having no events in this range can be estimated by $(1-0.042)^{528}=1.4 \times 10^{-10}$. Based on these simple calculations, one could justify the existence of a corner value: a characteristic scale that breaks the power-law behavior at the tail due to deviations from critical behavior or to finite-size effects [25]. However, this corner value would be only visible for the experiment at zero amplification; the same calculation for the experiment at $20 \mathrm{~dB}$ gives a probability of having no events above the maximum observed of 0.16 , which is not a small probability at all. Some recent works $[25,26]$ have reached similar conclusions for earthquakes and for complex networks. Thus it is important to identify and characterize deviations from power-law tails by means of appropriate statistical techniques.

\section{B. Global fit}

Once the particular fits have been performed, the ranges for which the power-law hypothesis cannot be rejected are known for each experiment $\left[A_{\min _{\mathrm{i}}}, A_{\text {max }_{\mathrm{i}}}\right]$ (see Table I). For each catalog $i$, we have $\hat{n}_{i}$ events that follow the distribution in Eq. (4) and we assume that there exists a global exponent $\hat{\alpha}_{g}$ that characterizes a global distribution that includes the power-law regimes for all the experiments.
Under these assumptions, for the particular case of amplitudes in $\mathrm{dB}$, the general log-likelihood function in Eq. (2) reads

$$
\begin{aligned}
\log \mathcal{L} & =\sum_{i=1}^{n_{\text {cat }}} \sum_{l=A_{\min _{i}}}^{A_{\max _{i}}} \omega_{i l} \log f_{\mathcal{Y}}^{(i)}[g(l)] \\
& =\sum_{i=1}^{n_{\text {cat }}} \sum_{l=A_{\min _{\mathrm{i}}}}^{A_{\max _{i}}} \omega_{i l} \log \left(\frac{2 \sinh [2.30(1-\alpha) \Delta / 20]}{V_{\max }^{1-\alpha}-V_{\min }^{1-\alpha}} \frac{1}{y_{l}^{\alpha-1}}\right)
\end{aligned}
$$

where $\omega_{i l}$ is the number of events with amplitude in $\mathrm{dB} l$ in the $i$ th experiment from a set of $n_{\text {cat }}$ catalogs $\left(\sum_{l=A_{\min _{\mathrm{i}}}}^{A_{\max _{\mathrm{i}}}} \omega_{i l}=\hat{n}_{i}\right)$.

After maximization of the log likelihood, the next step consists in determining whether the null hypothesis of considering a global exponent $\hat{\alpha}_{g}$ is compatible with the values of the particular fits shown in Table I. This procedure is explained in more detail in Appendix B. The global fit yields a global exponent $\hat{\alpha}_{g}=1.740 \pm 0.006$ with a $p$ value $=0.36$ for $\mathcal{N}=\sum_{i=1}^{n_{\text {cat }}} \hat{n}_{i}=31441$ events. Note that the value of the global exponent is in agreement with the weighted harmonic mean:

$$
\hat{\alpha}_{g}=1.740 \simeq 1+\frac{\mathcal{N}}{\sum_{i=1}^{n_{\text {cat }}} \frac{\hat{n}_{i}}{\hat{\alpha}_{i}-1}}=1.741
$$

(see Appendix D for a justification of this result). This procedure has been tested over simulated power-law data with the same parameters as in Table I, yielding acceptable $p$ values.

Figure 2 shows the global PDF for the amplitudes and the global fit. Observe how the global exponent is valid along 4.5 orders of magnitude, giving an unprecedented broad fitting range in amplitudes. The procedure to construct this aggregated histogram is explained in Appendix E. As the estimation of the probability density is done using bins [5], note that one can safely replace the unknown values of the random variable $\mathcal{V}$ by the known discretized values of $\mathcal{Y}$. The only requirement is that the width of the bins is not smaller than the discretization of $\mathcal{Y}$.

\section{ENERGIES}

\section{A. Particular fits}

Figure 3(a) shows the energy distributions for the complete dataset of all the experiments performed at different values of the preamplification. In contrast to the case of amplitudes, continuous values of the energy are collected. In order to 


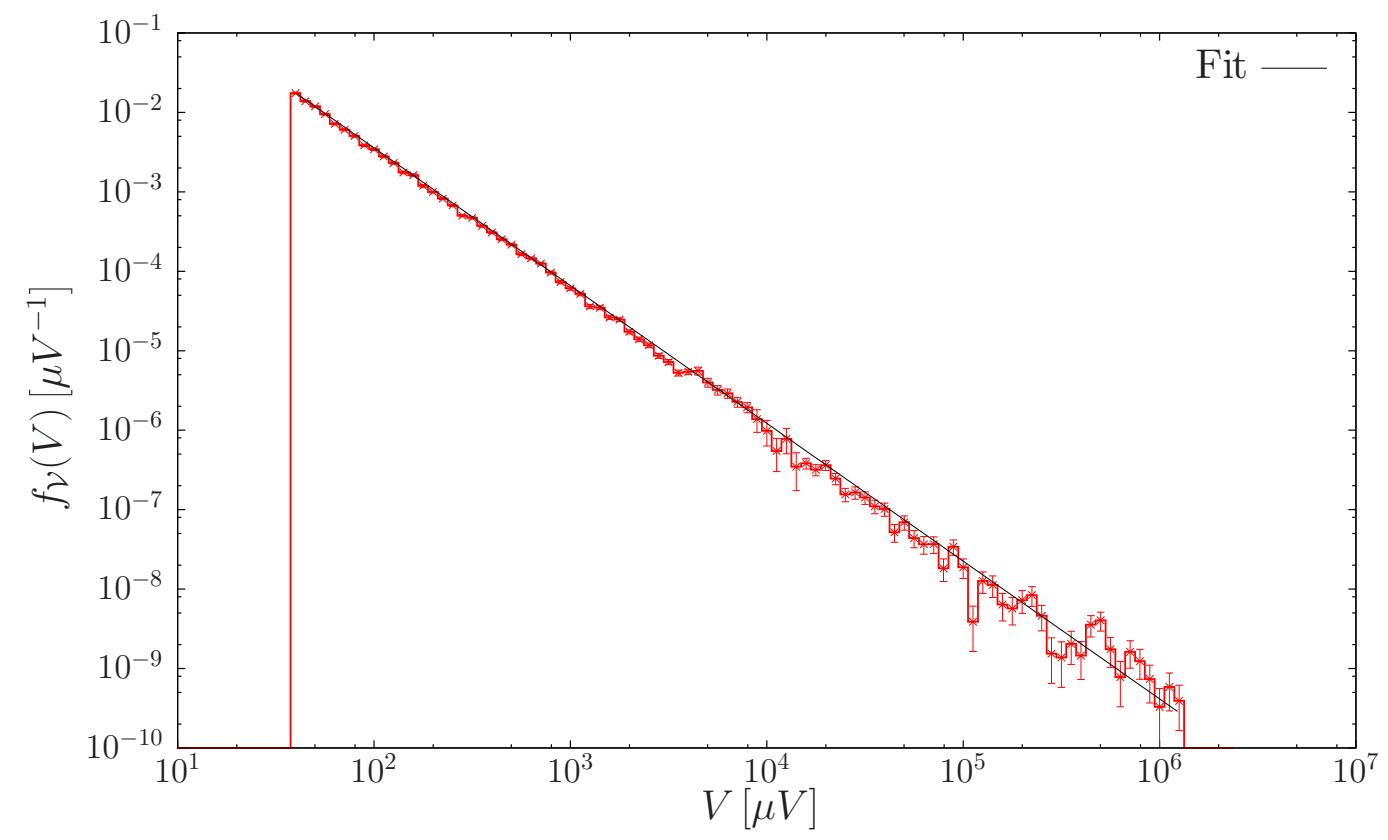

FIG. 2. Aggregated probability density of the avalanche amplitude together with a fit with exponent $\alpha_{g}=1.740 \pm 0.006, p$ value $=0.36$, and number of fitted data $\mathcal{N}=31441$. Error bars are estimated as the standard deviation for each bin [5]. Note that the fit ranges almost five decades, from $g(32-\Delta)$ to $g(122+\Delta)$.

properly represent the PDF, data have been binned using a convenient bin width (see Fig. 3). Due to the problems of saturation for large amplitudes and the presence of noise for small amplitudes, the energy corresponding to these events is not well estimated. In the following analysis we only consider events the amplitude of which lies in $\left[V_{\min _{i}}, V_{\text {max }_{i}}\right]$, where the ranges are those that have been found in the particular fits for amplitude PDF in Table I [see Fig. 3(b)]. We propose that the energy follows a truncated continuous power-law PDF:

$f_{\mathcal{E}}(E) d E=\frac{1-\epsilon}{E_{\max }^{1-\epsilon}-E_{\min }^{1-\epsilon}} E^{-\epsilon} d E$, where $E \in\left[E_{\min }, E_{\max }\right]$.

By fixing the values of the range $\left[E_{\min }, E_{\max }\right]$ we find the value of $\epsilon$ that maximizes the following log-likelihood function:

$$
\log \mathcal{L}=n \log \left(\frac{1-\epsilon}{E_{\max }^{1-\epsilon}-E_{\min }^{1-\epsilon}}\right)-\epsilon \sum_{j=1}^{n} \log E_{j},
$$

where $E_{j}$ are the particular values of the energy and $n$ is the number of data in $\left[E_{\min }, E_{\max }\right]$. Note that $E_{\min }$ and $E_{\max }$ do not have a direct correspondence with $V_{\min }$ and $V_{\max }$. Explicit details of this particular fit are given in Appendix A. The values of the fitted exponent $\hat{\epsilon}$ for different values of $E_{\text {min }}$ and $E_{\max }$ are shown in Appendix C by using MLE exponent maps [35]. In Table II we present fitting parameters when the minimum significance level is set at $p_{c}=0.20$. The values of the exponents are in rough agreement with the one reported in Ref. [19], in particular the value for $60 \mathrm{~dB}$.

\section{B. Global fit}

In order to write the log-likelihood function of the global fit, it turns out that each experiment contributes with $\hat{n}_{i}$ data which are distributed according to Eq. (8) in the range $\left[E_{\min _{\mathrm{i}}}, E_{\max _{\mathrm{i}}}\right]$ with a global exponent $\epsilon_{g}$ :

$$
\begin{aligned}
\log \mathcal{L}= & \mathcal{N} \log \left(1-\epsilon_{g}\right)-\epsilon_{g} \sum_{i=1}^{n_{\text {cat }}} \sum_{j=1}^{\hat{n}_{i}} \log E_{i j} \\
& -\sum_{i=1}^{n_{\text {cat }}} \hat{n}_{i} \log \left(E_{\max _{\mathrm{i}}}^{1-\epsilon_{g}}-E_{\min _{\mathrm{i}}}^{1-\epsilon_{g}}\right)
\end{aligned}
$$

where $\hat{n}_{i}$ is the number of data in the $i$ th catalog, $\mathcal{N}=\sum_{i}^{n_{\text {cat }}} \hat{n}_{i}$, and $E_{i j}$ are the values of the energy in each power-law regime $i$. The values of the ranges $\left[E_{\min _{\mathrm{i}}}, E_{\text {max }_{\mathrm{i}}}\right]$ are taken from the particular fits in Table II. Details of the goodness-of-fit test for this global fit are explained in Appendix B. By considering the particular ranges shown in Table II, the global fit of the energy exhibits an exponent $\hat{\epsilon}_{g}=1.352 \pm 0.004\left(\mathcal{N}=\sum_{i=1}^{n_{\text {cat }}}, \hat{n}_{i}=\right.$ 21836 ) along more than nine decades. As it happens for the case of the global amplitude distribution, the value of the global exponent is in agreement with the weighted harmonic mean:

$$
\hat{\epsilon}_{g}=1.352 \simeq 1+\frac{\mathcal{N}}{\sum_{i=1}^{n_{\text {cat }}} \frac{\hat{n}_{i}}{\hat{\epsilon}_{i}-1}}=1.347 .
$$

As we have mentioned, this result is justified in Appendix D.

Nevertheless, this global fit does not fulfil the goodnessof-fit test and the null hypothesis $H_{0}$ that all the catalogs share a common exponent $\hat{\epsilon}_{g}$ is rejected. In Fig. 4 we show the aggregated empirical probability density for the energy of the AE events. This histogram has been constructed following the procedure explained in Appendix E. Simulated data with the same parameters as in Table II also yield the same rejection of the null hypothesis. We have performed the same analysis without the restriction of just considering events with 

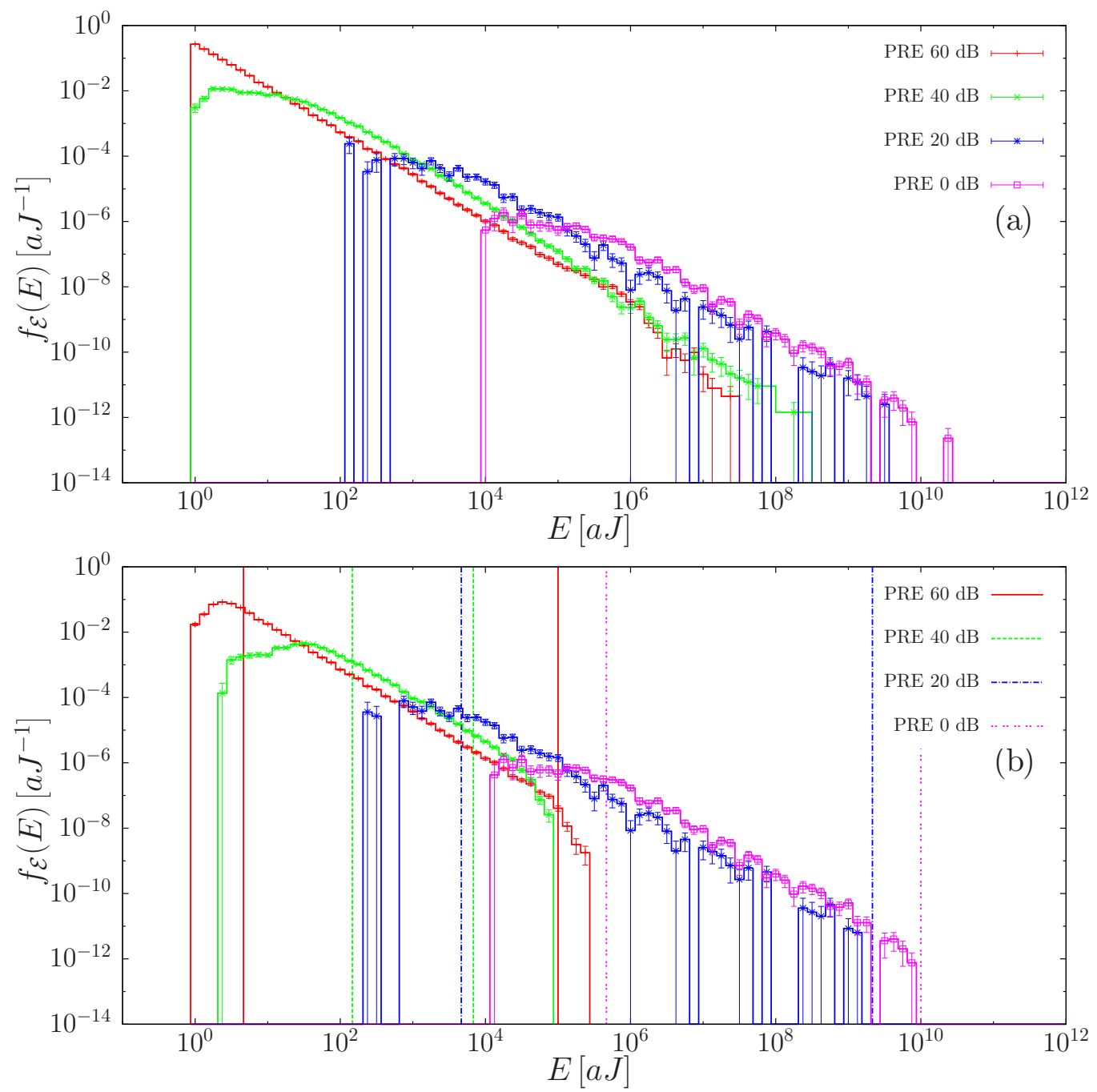

FIG. 3. Estimated energy PDFs for the different experiments performed at different preamplifications (PRE). (a) Complete datasets. (b) Events with amplitude in the power-law range $V \in\left[V_{\min _{i}}, V_{\text {max }_{\mathrm{i}}}\right]$. Vertical bars of the same color as the PDFs and same line style between them correspond to the power-law ranges listed in Table II. Error bars are estimated as the standard deviation for each bin [5].

amplitude $V \in\left[V_{\min _{\mathrm{i}}}, V_{\text {max }_{\mathrm{i}}}\right]$ or of sparing some catalogs. In all the cases, the rejection of the null hypothesis occurs.

We can speculate about different distortion factors that could explain these results.

(1) The first effect comes from a biased measurement of the energy caused by the dependence of the measured event duration with the detection threshold. The higher the threshold, the shorter the duration and the lower the energy since this corresponds to the integrated (squared) AE signal along the registered duration. This fact does not occur for the case of the amplitudes, since these are independent of the value of the threshold.

(2) A second explanation could be associated to the fact that for higher thresholds (lower amplifications) some tails of

TABLE II. Fitted parameters for Eq. (8) for each experiment. $\hat{\epsilon}$ corresponds to the fitted exponent in the range $\left[E_{\min }, E_{\max }\right]$ for which the goodness-of-fit test exceeds the significance level $p_{c}=0.2 . \hat{n}$ is the number of data entering into the fit and $N$ is the total number of events in the dataset. Columns 3 and 4 refer to the minimum and maximum energy corresponding to amplitude in the range $\left[V_{\min _{\mathrm{i}}}, V_{\max _{\mathrm{i}}}\right]$. Error bars of the exponent correspond to the standard deviation of the MLE.

\begin{tabular}{|c|c|c|c|c|c|}
\hline $\operatorname{PRE}(\mathrm{dB})$ & $\hat{\epsilon}$ & $E_{\min }(\mathrm{aJ})$ & $E_{\max }(\mathrm{aJ})$ & $\hat{n}(N)$ & $p$ \\
\hline 60 & $1.360 \pm 0.004$ & $4.642(1.001)$ & $10^{5}\left(3.005 \times 10^{5}\right)$ & $16342(21414)$ & 0.43 \\
\hline 40 & $1.32 \pm 0.02$ & $146.780(3.133)$ & $6.812 \times 10^{3}\left(9.378 \times 10^{4}\right)$ & 4814 (9146) & 0.32 \\
\hline 20 & $1.29 \pm 0.02$ & $4641.589(270.446)$ & $2.15 \times 10^{9}\left(1.588 \times 10^{9}\right)$ & $284(353)$ & 0.33 \\
\hline 0 & $1.27 \pm 0.02$ & $4.642 \times 10^{5}\left(1.545 \times 10^{4}\right)$ & $10^{10}\left(9.156 \times 10^{9}\right)$ & $396(528)$ & 0.55 \\
\hline Global & $1.352 \pm 0.004$ & $4.642(1.001)$ & $10^{10}\left(9.156 \times 10^{9}\right)$ & $21836(31441)$ & 0 \\
\hline
\end{tabular}




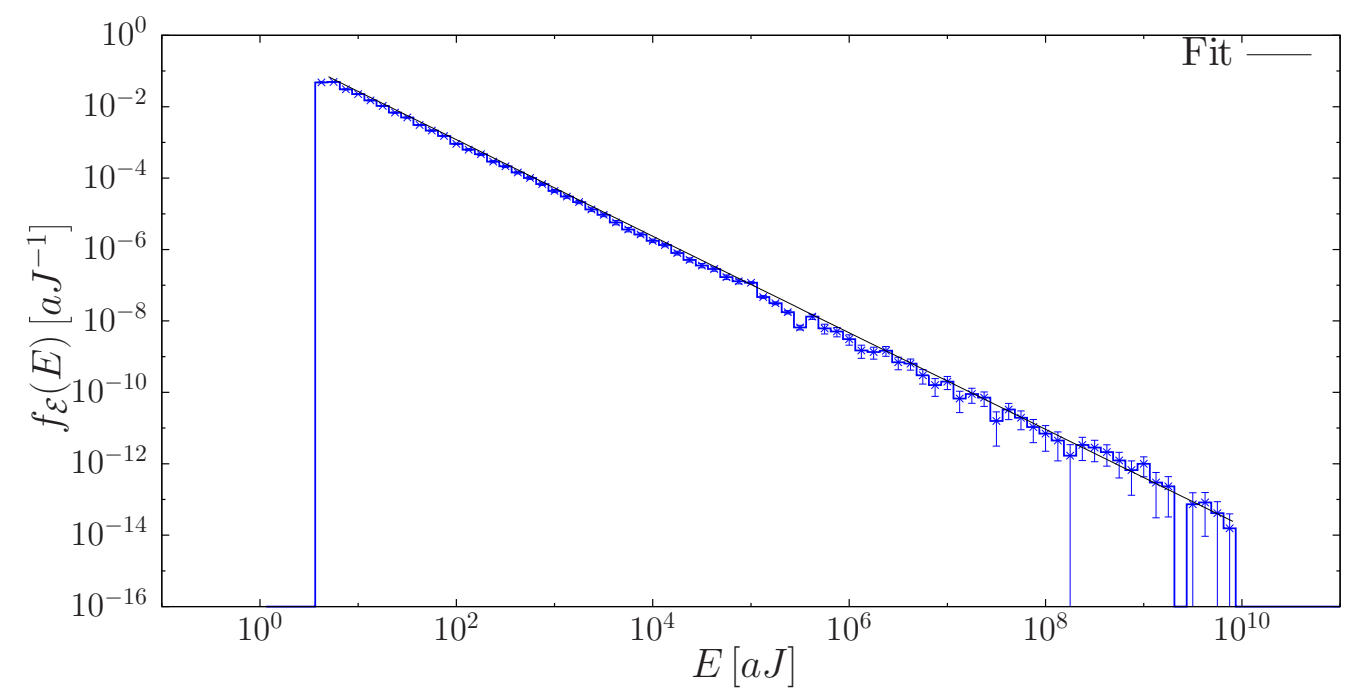

FIG. 4. Aggregated empirical energy PDF for all the experiments. Error bars are estimated as the standard deviation for each bin [5]. The black solid line corresponds to the fit of the truncated power law with exponent $\hat{\epsilon}_{g}=1.352$ in the range $\left[4.642 \mathrm{aJ}, 10^{10}\right.$ aJ].

long fluctuating events are separated into different events, thus deforming the statistics.

(3) Third, one could also imagine other experimental factors due to distortions created by the fact that $\mathrm{AE}$ transducers have a certain relaxation time and/or a preferred resonant frequency.

(4) Fourth, there could be distortions in the duration of the signals due to the propagation of the ultrasounds in the sample.

(5) Finally, the distortion could be associated to real physical mechanisms in the source of AE events, like frictional losses that affect signals with different energies in a different way.

Detailed discussion of these effects clearly exceeds the goals of the present paper.

\section{CONCLUSIONS}

In this paper we have presented a methodology to estimate a global exponent for the PDF of certain avalanche properties by using different catalogs of events. This methodology has been applied to amplitudes and energies in AE avalanches recorded during different compression experiments of porous glasses. For the case of the amplitude PDF, a global exponent has been found spanning 4.5 orders of magnitude. To our knowledge, this is the broadest fitting range that has been found for the amplitude distribution of AE events. For the case of the energies, we graphically obtain an apparent power law spanning 9.5 decades. However, precise statistical analysis shows that the hypothesis of the existence of a global exponent for the energy does not hold. Experimental limitations due to the set of thresholds and definitions of AE avalanches could justify the rejection of the null hypothesis. We expect this methodology to be useful to broaden the range of power-law fits in different distributions that appear in experimental works in condensed matter physics and in other complex systems, for instance, earthquakes.

\section{ACKNOWLEDGMENTS}

The research leading to these results has received founding from "La Caixa" Foundation. V.N. acknowledges financial support from the Spanish Ministry of Economy and Competitiveness (Spain), through the "María de Maeztu" Programme for Units of Excellence in R\&D (Grant No. MDM-2014-0445). We also acknowledge financial support from the Spanish Ministry of Economy and Competitiveness under Grants No. FIS2015-71851-P and No. MAT2013-40590-P and Proyecto Redes de Excelencia Grant No. MAT2015-69777-REDT, and from Agència de Gestió d'Ajuts Universitaris i de Recerca (AGAUR) under Grant No. 2014SGR-1307.

\section{APPENDIX A: FITTING A TRUNCATED POWER-LAW DISTRIBUTION TO A DATASET}

In this Appendix we present the procedure to fit via MLE of a probability mass function (PMF) or a PDF to an empirical dataset of $N$ values $\{x\}$. The procedure is essentially the same as the one in Ref. [5], but we summarize it here for completeness. The PMF or PDF $f(x ; \gamma, a, b)$ is characterized by a parameter $\gamma$ (exponent) and two values for the lower $a$ and upper $b$ truncations. The first step consists in writing the log-likelihood function:

$$
\log \mathcal{L}=\log \prod_{i=1}^{n} f\left(x_{i} ; \gamma, a, b\right)=\sum_{i=1}^{n} \log f\left(x_{i} ; \gamma, a, b\right),
$$

where $n$ is the number of data between $a$ and $b$. Depending on whether we are dealing with a PMF or a PDF, one has to use a different expression of $f(x ; \gamma, a, b)$. Since the values of the fitting range $a$ and $b$ are not known $a$ priori, 20 partitions per decade in log scale are used in order to sweep all the possible intervals $[a, b]$ for the amplitude and six partitions per decade for the energy. The value of the empirical exponent $\gamma$ that maximizes Eq. (A1) is computed for each interval by means of the function optimize, which is already implemented in $\mathrm{R}$ programming language $[38,39]$. Once this value is found, one has to determine through a statistical test whether the fit is "acceptable" or not. The null hypothesis states that data $\{x\}$ are sampled from a truncated power-law distribution with exponent $\gamma$. In order to check whether this null hypothesis is 
rejected or not, the Kolmogorov-Smirnov (KS) distance is used [40]. The KS statistic measures the maximum distance between the empirical cumulative distribution function $(\mathrm{CDF}) F_{e}$ (the subscript $e$ refers to empirical) and the analytic expression $F$ :

$$
d_{e}=\max \left|F_{e}(x ; a, b)-F(x ; \gamma, a, b)\right| .
$$

Once the value of this distance is known, it is necessary to state whether its value is large or not compared to those $\left\{d_{\text {sim }}\right\}$ found when the original data are really sampled from a truncated power-law distribution with exponent $\gamma$. In order to perform this estimation, a number $N_{\text {sim }}\left(N_{\text {sim }}=1000\right)$ of simulations of $n$ values sampled from $f(x ; \gamma, a, b)$ is done. A fitted exponent $\gamma_{\text {sim }}$ for simulated data is computed by maximizing Eq. (A1) and a KS distance $d_{\text {sim }}$ is found for each simulated dataset. The $p$ value of the test is estimated as the fraction of observations where $d_{e} \leqslant d_{\text {sim. }}$. If the $p$ value of the fit exceeds a certain threshold $p_{c}$ then one considers that the null hypothesis is accepted (in the sense that it cannot be rejected). From all the possible intervals the $p$ value of which exceeds $p_{c}$, the one with the largest number of data is chosen to yield the right power-law range with exponent $\hat{\gamma}$.

\section{APPENDIX B: GOODNESS-OF-FIT TEST FOR GLOBAL DISTRIBUTIONS}

Determining whether the null hypothesis of considering a global exponent $\Gamma$ is compatible with the values of the particular fits has some differences with respect to the case explained in Appendix A. In this Appendix we detail the goodness-of-fit test that has been applied to the global fit. First of all, we need to redefine the KS distance in this case. When the value of the global exponent $\hat{\Gamma}$ has been found, one can picture that each dataset that contributes to the global PDF has been fitted with a global exponent $\hat{\Gamma}$ in their particular ranges $\left[a_{i}, b_{i}\right]\left(i=1, \ldots, n_{\text {cat }}\right)$. Therefore $n_{\text {cat }} \mathrm{KS}$ distances can be found by

$$
D_{e, i}=\max \left|F_{e, i}\left(x ; a_{i}, b_{i}\right)-F_{i}\left(x ; \hat{\Gamma}, a_{i}, b_{i}\right)\right|,
$$

where the subindex $i$ refers to the $i$ th catalog, $F_{e}$ is the empirical $\mathrm{CDF}$, and $F$ is the analytical CDF. In order to compute a global KS distance, we perform the following summation:

$$
D=\sum_{i=1}^{n_{\text {cat }}} \sqrt{\hat{n}_{i}} D_{e, i},
$$

where the factors $\sqrt{\hat{n}_{i}}$ are due to the scaling of the KS distance with the number of data [40]. Once the empirical KS distance is found, one needs to determine whether this distance is big or small in relation to the KS distance found for data sampled from a PDF with the same parameters $a_{i}, b_{i}, \hat{\Gamma}$, and $\hat{n}_{i}$. Data are generated in the range given by the particular fit of the $i$ th catalog with probability $q_{i}=\hat{n}_{i} / \mathcal{N}$, where $\mathcal{N}=\sum_{i=1}^{\mathrm{n}_{\text {cat }}} \hat{n}_{i}$. Note that the particular number in each simulated dataset is not necessarily the empirical one $\hat{n}_{i}$ but the total number of data $\mathcal{N}$ is maintained. Hence, one needs a first random number to choose the dataset $i$ and therefore the range $\left[a_{i}, b_{i}\right]$ and a second one to generate the random truncated power-law number in that range with exponent $\hat{\Gamma}$ [5]. When $N$ events have been generated according to this procedure, one finds the global exponent $\hat{\Gamma}_{\text {sim }}$ by maximizing the global log likelihood and computes the global KS distance $D_{\text {sim }}$ for simulated data. By performing several realizations of the previous procedure, one can estimate the $p$ value of the fit by computing the fraction of simulated datasets where the simulated global KS distance is larger than the empirical one.

\section{APPENDIX C: MLE EXPONENT MAPS}

In order to complement the information of the particular fits, we show the MLE exponent maps. These maps show the value of the exponent of a truncated power law as a function
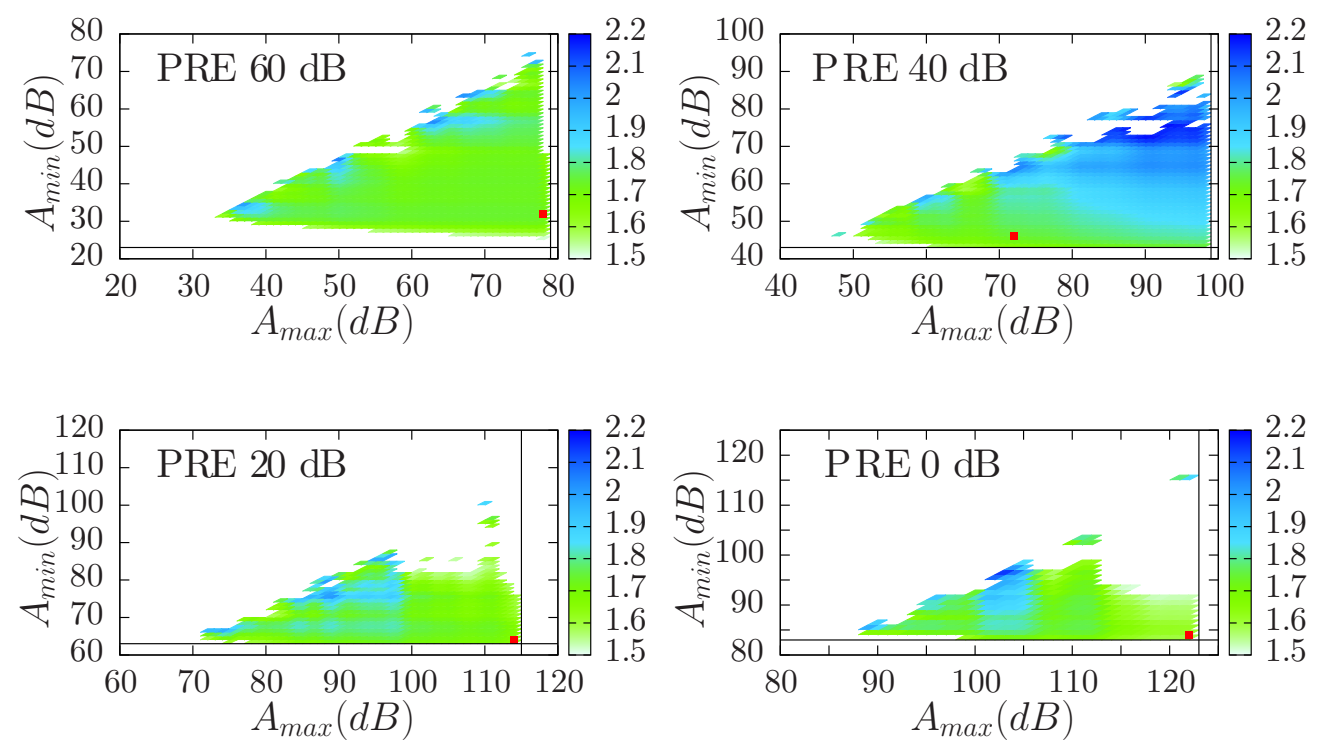

FIG. 5. MLE exponent maps for the distribution of amplitudes in $\mathrm{dB}$ of every experiment. Red-squared points indicate the values of the exponent and the upper and lower truncations found in Table I in the main text. White gaps correspond to regions that are out of the color range that appears at the right of each map. Black solid lines correspond to the maximum and minimum values in the sample. 

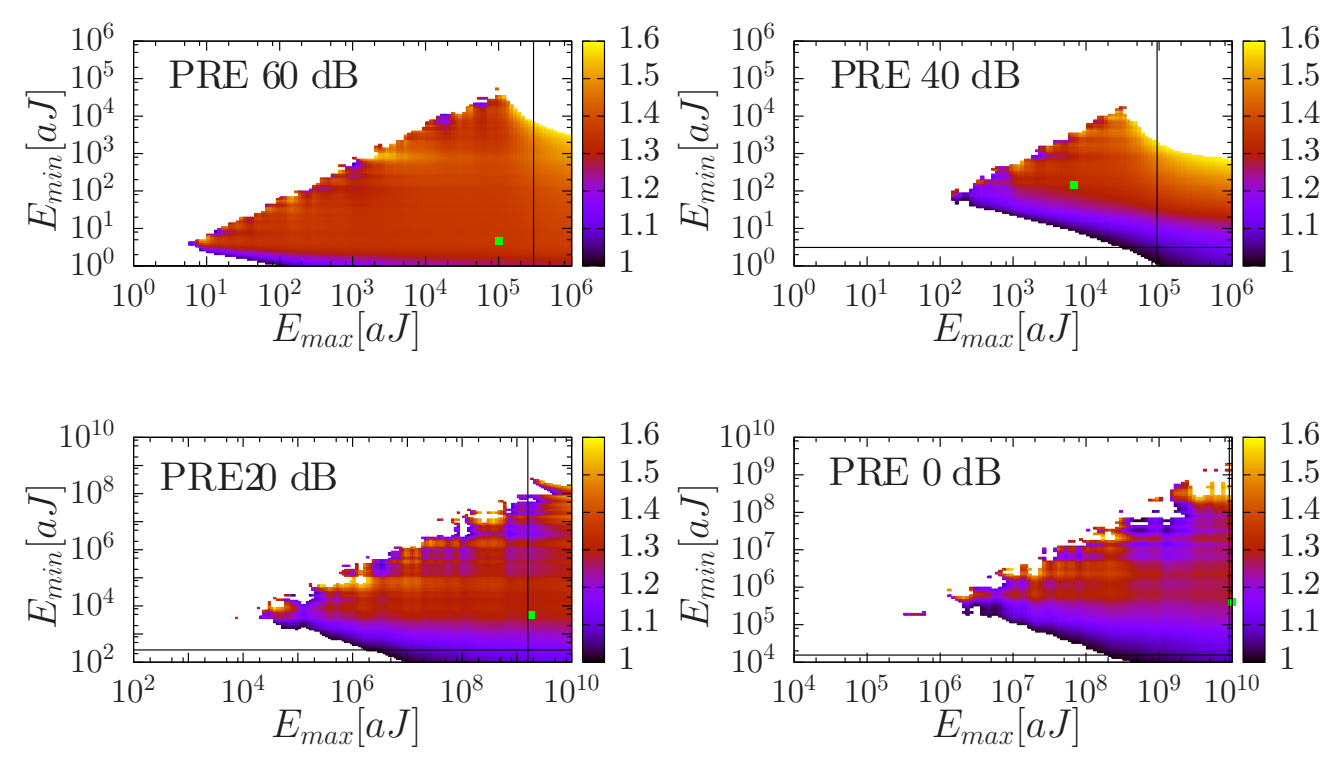

FIG. 6. MLE exponent maps for the energy for each experiment. Green-squared points indicate the values of the exponent and the upper and lower truncations found in Table II in the main text. White gaps correspond to regions that are out of the color range that appears at the right of each map. Black solid lines correspond to the maximum and minimum values in the sample.

of the values of the upper and lower truncations. This kind of representation is useful since it gives information about how stable is the value of the exponent as the truncations of the power-law fit change. In Figs. 5 and 6 we show the MLE exponent maps for the case of the amplitudes in $\mathrm{dB}$ and energy, respectively. Due to the condition that the variable $\mathcal{X}$ must fulfil $x_{\min } \leqslant x_{\max }$ for the upper and lower truncations, the maps exhibits a triangular shape. The points in each map show the upper and lower truncations as well as the exponent of the fit which has been done according to the fitting procedure explained in Appendix A. As it can be observed, this fit is placed in uniform-colored regions of the map.

\section{APPENDIX D: GLOBAL EXPONENT}

In this Appendix we expand Eq. (2) for a nontruncated power-law PDF in order to relate the global exponent with the particular ones. Let us consider $n_{\text {cat }}$ catalogs with $\hat{n}_{i}$ events characterized by a variable $\mathcal{X}$. We propose that for the $i$ th catalog data follow a power law with exponent $\gamma_{i}$ from a lower cutoff $a_{i}$ to $\infty$ :

$$
f_{\mathcal{X}}^{(i)}(x)=\frac{\gamma_{i}-1}{a_{i}^{1-\gamma_{i}}} x^{-\gamma_{i}} .
$$

By finding the value of the particular exponent that maximizes the log-likelihood expression in Eq. (A1) we obtain [4,5]

$$
\hat{\gamma}_{i}=1+\frac{\hat{n}_{i}}{\sum_{j=1}^{\hat{n}_{i}} \log \left(x_{j} / a\right)} .
$$

Now we consider that data in these $n_{\text {cat }}$ catalogs follow PDFs with different cutoffs $a_{i}$ but sharing a global exponent $\Gamma$. If we bring these PDFs to the global log likelihood of Eq. (2), we obtain

$$
\begin{aligned}
\log \mathcal{L}= & \sum_{i=1}^{n_{\text {cat }}} \hat{n}_{i} \log (\Gamma-1)+\sum_{i=1}^{n_{\text {cat }}} \hat{n}_{i}(\Gamma-1) \log a_{i} \\
& -\Gamma \sum_{i=1}^{n_{\text {cat }}} \sum_{j=1}^{\hat{n}_{i}} \log x_{j} .
\end{aligned}
$$

By deriving this expression with respect to $\Gamma$ and making the result equal to zero, we can obtain the value of the global exponent that maximizes the log likelihood:

$$
\hat{\Gamma}=1+\frac{\mathcal{N}}{\sum_{i=1}^{n_{\text {cat }}} \sum_{j=1}^{\hat{n}_{i}} \log \left(x_{j} / a_{i}\right)} .
$$

We can relate this global exponent $\Gamma$ with the particular exponents from Eq. (D2) leading to

$$
\frac{\mathcal{N}}{\hat{\Gamma}-1}=\sum_{i=1}^{n_{\text {cat }}} \frac{\hat{n}_{i}}{\hat{\gamma}_{i}-1} .
$$

Hence, for the case of nontruncated power-law PDFs, the global exponent is related to the weighted harmonic mean of $\gamma_{i}-1$. For truncated power laws, if the range is sufficiently big, one expects this result to provide a good approximation.

\section{APPENDIX E: AGGREGATED GLOBAL HISTOGRAM}

We would like to represent a global histogram of a certain variable $\mathcal{X}$ that is sampled from $n_{\text {cat }}$ datasets, each dataset in a different domain (or different support, in probabilistic language), but assuming a common underlying distribution. Obviously, the difference in the domains will lead to bumps and irregularities if all data are taken together into a simple histogram. As an example, two overlapping histograms are shown in Fig. 7 sampled from a uniform distribution (the total domain 


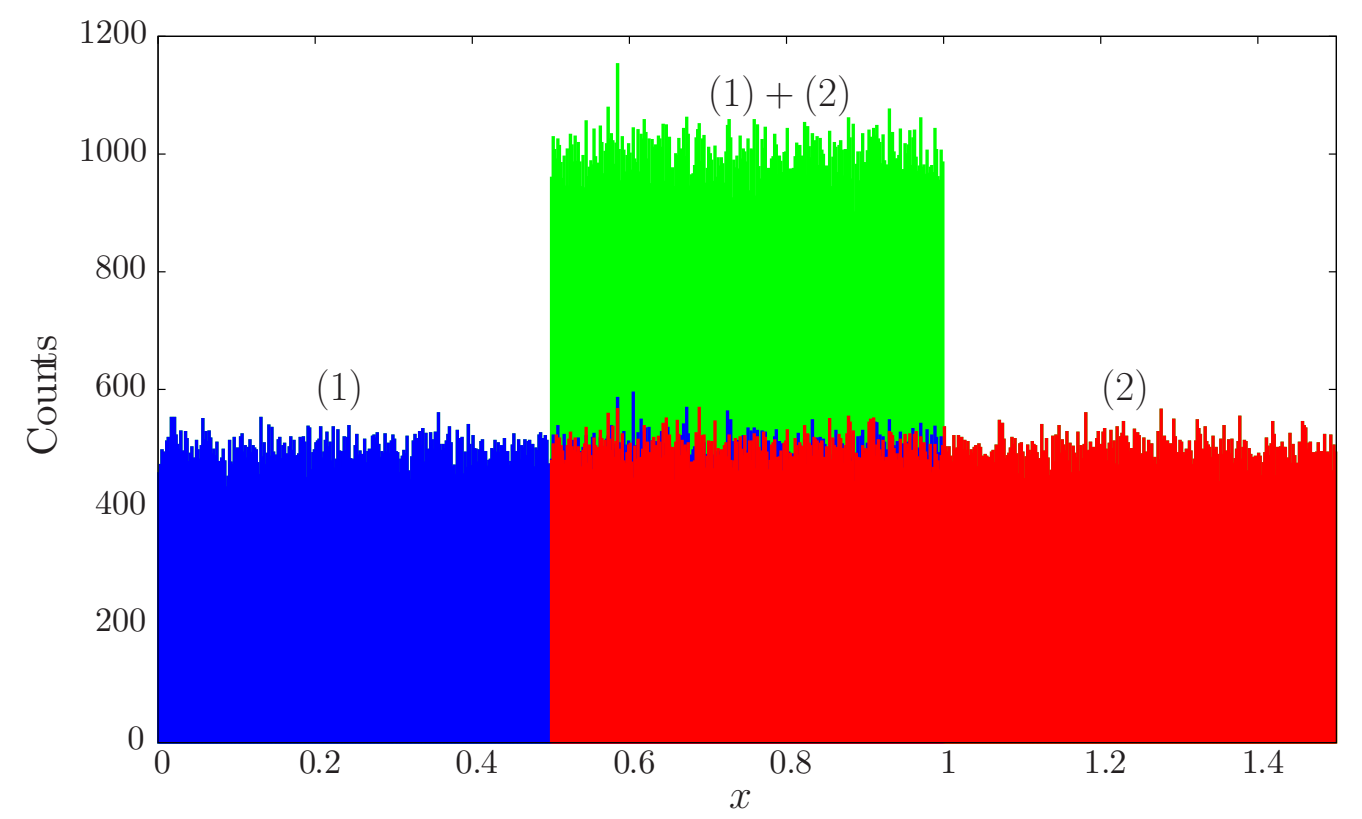

FIG. 7. Histogram 1 of $2.5 \times 10^{4}$ numbers sampled from a uniform distribution in $(0,1)$, together with the histogram 2 of $2.5 \times 10^{4}$ numbers sampled from another uniform distribution in $(0.5,1.5)$. The (naive) sum of these histograms $(1+2)$ is also shown, where an excess of counts can be appreciated in the intersection region.

of which is supposedly unknown), restricted to the interval $(0,1)$ for histogram 1 and to $(0.5,1.5)$ for histogram 2 . We are going to construct a single histogram without the anomalies that arise in the different regions if a naive procedure is used. Our procedure is independent of the shape of the histogram (a convenient change of variable can transform any distribution into a uniform one, without affecting the procedure; this is the reason to use the uniform example in Fig. 7).

Let us start with two datasets, 1 and 2, and let $m_{1}$ be the number of data from dataset 1 overlapping with dataset 2 and let $m_{2}$ be the number of data from dataset 2 overlapping with dataset 1 , where by overlapping we mean that the points belong to the intersection of both domains. Instead of associating a data point to one count we consider that each data point contributes to the global histogram proportionally to the inverse of the "sample intensity," to be concrete, nonoverlapping data from datasets 1 and 2 contributing to the global histogram with $\frac{1}{m_{1}}$ and $\frac{1}{m_{2}}$, respectively, whereas the overlapping data contribute with $\frac{1}{m_{1}+m_{2}}$. The latter assumption comes from the fact that if we used $\frac{1}{m_{i}}$ for the overlapping data from dataset 1 we would obtain a histogram twice as high as it should be (due precisely to the overlapping). Weighting each contribution by $\frac{m_{i}}{m_{1}+m_{2}}$ leads to the $\frac{1}{m_{1}+m_{2}}$ value. After this rescaling, datasets 1 and 2 are assembled in a single (smooth) histogram $1+2$.

In order to add a new dataset 3 , we proceed recursively. We need to evaluate the contribution of $1+2$ that overlaps with dataset 3 . We define $m_{12}$ as the number of data of dataset 1 or 2 overlapping with dataset 3 , and we define the weight $\rho_{12}$ of this overlapping region as

$$
\rho_{12}=\underbrace{\frac{1}{m_{1}}+\cdots+\frac{1}{m_{1}}+\frac{1}{\left(m_{1}+m_{2}\right)}+\cdots+\frac{1}{\left(m_{1}+m_{2}\right)}+\frac{1}{m_{2}}+\ldots \frac{1}{m_{2}}}_{m_{12} \text { terms }},
$$

where the three different terms in the sum come from the data in dataset 1 that do not overlap with dataset 2, the data in dataset 1 that overlap with dataset 2 or vice versa, and the data in dataset 2 that do not overlap with dataset 1 , respectively (and with all of them overlapping with dataset 3 ). In order to compare properly the histogram $1+2$ with dataset 3 , the former should come with a weight $m_{12}$ (instead of $\rho_{12}$ ), so we rescale the former by the factor $\frac{m_{12}}{\rho_{12}}$. In this way, the histogram from $1+2$ turns out to have the different contributions $\frac{m_{12}}{m_{1} \rho_{12}}, \frac{m_{12}}{\left(m_{1}+m_{2}\right) \rho_{12}}$, and $\frac{m_{12}}{m_{2} \rho_{12}}$ (depending on which part of the domain each data point comes from). The next step consists in applying the original procedure to $1+2$ and dataset 3 , thus rescaling in each case by $\frac{1}{m_{12}}, \frac{1}{m_{12}+m_{3}}$, or $\frac{1}{m_{3}}$, where $m_{3}$ refers to the number of data from dataset 3 overlapping with $1+2$ (i.e., with dataset 1 or 2 ). To be precise, data points from the already assembled histogram $1+2$ not overlapping with dataset 3 contribute introducing the extra factor $\frac{1}{m_{12}}$, yielding

$$
\frac{1}{m_{1} \rho_{12}}, \frac{1}{\left(m_{1}+m_{2}\right) \rho_{12}}, \frac{1}{m_{2} \rho_{12}}
$$


for each case; the overlapping data points contribute $\frac{1}{\left(m_{12}+m_{3}\right)}$, yielding

$$
\frac{m_{12}}{m_{1}\left(m_{12}+m_{3}\right) \rho_{12}}, \quad \frac{m_{12}}{\left(m_{1}+m_{2}\right)\left(m_{12}+m_{3}\right) \rho_{12}}, \frac{m_{12}}{m_{2}\left(m_{12}+m_{3}\right) \rho_{12}}
$$

if the data point came from dataset 1 or 2 , and $\frac{1}{m_{12}+m_{3}}$ if they come from dataset 3 ; nonoverlapping data points from the new dataset 3 contribute $\frac{1}{m_{3}}$.

One can iterate this procedure in order to add as many datasets as necessary. In any case, note that one can only add datasets that overlap, as the overlapping region is used as a way of "calibration." So, the order in which the aggregation is performed is important (in our catalogs of amplitudes, the datasets for 0 and $60 \mathrm{~dB}$ cannot be aggregated directly, as they do not overlap, but they can be aggregated after one of them is previously aggregated with the datasets for 20 and $40 \mathrm{~dB}$ ). In general, in order to have an estimation of a PDF, rescaled counts must be divided by the bin width of each bin and, secondly, the resulting histogram has to be divided by its integral over the full domain in order to fulfil normalization. For a probability mass function, one has to divide by the sum of the histogram values. Error bars are subject to the same rescaling transformations.
[1] J. P. Sethna, K. A. Dahmen, and C. R. Myers, Crackling noise, Nature (London) 410, 242 (2001).

[2] E. K. H. Salje and K. A. Dahmen, Crackling noise in disordered materials, Annu. Rev. Condens. Matter Phys 5, 233 (2014).

[3] P. Bak, How Nature Works: The Science of Self-Organized Criticality (Copernicus, New York, 1996).

[4] A. Clauset, C. R. Shalizi, and M. E. J. Newman, Power-law distributions in empirical data, SIAM Rev. 51, 661 (2009).

[5] A. Deluca and Á. Corral, Fitting and goodness-of-fit test of nontruncated and truncated power-law distributions, Acta Geophys. 61, 1351 (2013).

[6] K. Christensen and N. R. Moloney, Complexity and Criticality (Imperial College, London, 2005).

[7] N. Friedman, S. Ito, Braden A. W. Brinkman, M. Shimono, R. E. Lee. DeVille, K. A. Dahmen, J. M. Beggs, and T. C. Butler, Universal Critical Dynamics in High Resolution Neuronal Avalanche Data, Phys. Rev. Lett. 108, 208102 (2012).

[8] S. Papanikolaou, F. Bohn, R. L. Sommer, G. Durin, S. Zapperi, and J.P. Sethna, Universality beyond power laws and the average avalanche shape, Nat. Phys. 7, 316 (2011).

[9] J. T. Uhl, S. Pathak, D. Schorlemmer, X. Liu, R. Swindeman, B. A. W. Brinkman, M. LeBlanc, G. Tsekenis, N. Friedman, R. Behringer, D. Denisov, P. Schall, X. Gu, W. J. Wright, T. Hufnagel, A. Jennings, J. R. Greer, P. K. Liaw, T. Becker, G. Dresen, and K. A. Dahmen, Universal quake statistics: From compressed nanocrystals to earthquakes, Sci. Rep. 5, 16493 (2015).

[10] D. Avnir, O. Biham, D. Lidar, and O. Malcai, Is the geometry of nature fractal? Science 279, 39 (1998).

[11] O. Malcai, D. Lidar, O. Biham, and D. Avnir, Scaling range and cutoffs in empirical fractals, Phys. Rev. E 56, 2817 (1997).

[12] E. Vives, J. Ortín, L. Mañosa, I. Ràfols, R. Pérez-Magrané, and A. Planes, Distributions of Avalanches in Martensitic Transformations, Phys. Rev. Lett. 72, 1694 (1994).

[13] A. Petri, G. Paparo, A. Vespignani, A. Alippi, and M. Costantini, Experimental Evidence for Critical Dynamics in Microfracturing Processes, Phys. Rev. Lett. 73, 3423 (1994).

[14] E. Vives, I. Ràfols, L. Mañosa, J. Ortín, and A. Planes, Statistics of avalanches in martensitic transformations. I. Acoustic emission experiments, Phys. Rev. B 52, 12644 (1995).

[15] L. Carrillo, L. Mañosa, J. Ortín, A. Planes, and E. Vives, Experimental Evidence for Universality of Acoustic Emission
Avalanche Distributions During Structural Transitions, Phys. Rev. Lett. 81, 1889 (1998).

[16] J. Weiss, T. Richeton, F. Louchet, F. Chmelik, P. Dobron, D. Entemeyer, M. Lebyodkin, T. Lebedkina, C. Fressengeas, and R. J. McDonald, Evidence for universal intermittent crystal plasticity from acoustic emission and high-resolution extensometry experiments, Phys. Rev. B 76, 224110 (2007).

[17] J. Weiss, F. Lahaie, and J. R. Grasso, Statistical analysis of dislocation dynamics during viscoplastic deformation from acoustic emission, J. Geophys. Res. Solid Earth 105, 433 (2000).

[18] C. Miguel, A. Vespignani, S. Zapperi, J. Weiss, and J. R. Grasso, Complexity in dislocation dynamics: Experiments, Mater. Sci. Eng. A 309, 360 (2001).

[19] J. Baró, Á. Corral, X. Illa, A. Planes, E. K. H. Salje, W. Schranz, D. E. Soto-Parra, and E. Vives, Statistical Similarity Between the Compression of a Porous Material and Earthquakes, Phys. Rev. Lett. 110, 088702 (2013).

[20] G. F. Nataf, P. O. Castillo-Villa, J. Baró, X. Illa, E. Vives, A. Planes, and E. K. H. Salje, Avalanches in compressed porous SiO2-based materials, Phys. Rev. E 90, 022405 (2014).

[21] V. Navas-Portella, Á. Corral, and E. Vives, Avalanches and force drops in displacement-driven compression of porous glasses, Phys. Rev. E 94, 033005 (2016).

[22] T. Mäkinen, A. Miksic, M. Ovaska, and M. J. Alava, Avalanches in Wood Compression, Phys. Rev. Lett. 115, 055501 (2015).

[23] H. V. Ribeiro, L. S. Costa, L. G. A. Alves, P. A. Santoro, S. Picoli, E. K. Lenzi, and R. S. Mendes, Analogies Between the Cracking Noise of Ethanol-Dampened Charcoal and Earthquakes, Phys. Rev. Lett. 115, 025503 (2015).

[24] S. Lherminier, R. Planet, G. Simon, K. J. Måløy, L. Vanel, and O. Ramos, A granular experiment approach to earthquakes, Revista Cubana de Física 33, 55 (2015).

[25] I. Serra and Á. Corral, Deviation from power law of the global seismic moment distribution, Sci. Rep. 7, 40045 (2017).

[26] A. D. Broido, and A. Clauset, Scale-free networks are rare, arXiv:1801.03400 (2018).

[27] A. Corral and F. Font-Clos, Criticality and self-organization in branching processes: Application to natural hazards, in SelfOrganized Criticality Systems, edited by M. Aschwanden (Open Academic, Berlin, 2013), pp. 183-228.

[28] A. Corral, R. Garcia-Millan, N. R. Moloney, and F. Font-Clos (unpublished). 
[29] A. Deluca, P. Puig, and Á. Corral, Testing universality in critical exponents: The case of rainfall, Phys. Rev. E 93, 042301 (2016).

[30] PCI-2, A PCI-based two-channel AE board and system, Physical Acoustics.

[31] M. Paczuski, S. Boettcher, and M. Baiesi, Interoccurrence Times in the Bak-Tang-Wiesenfeld Sandpile Model: A Comparison with the Observed Statistics of Solar Flares, Phys. Rev. Lett. 95, 181102 (2005).

[32] A. Deluca, N. R. Moloney, and Á. Corral, Data-driven prediction of thresholded time series of rainfall and self-organized criticality models, Phys. Rev. E 91, 052808 (2015).

[33] F. Font-Clos, G. Pruessner, N. R. Moloney, and A. Deluca, The perils of thresholding, New J. Phys. 17, 043066 (2015).

[34] S. Janićević, L. Laurson, K. J. Måløy, S. Santucci, and M. J. Alava, Interevent Correlations from Avalanches Hiding Below the Detection Threshold, Phys. Rev. Lett. 117, 230601 (2016).
[35] J. Baró and E. Vives, Analysis of power-law exponents by maximum-likelihood maps, Phys. Rev. E 85, 066121 (2012).

[36] M. Koslowski, R. LeSar, and R. Thomson, Avalanches and Scaling in Plastic Deformation, Phys. Rev. Lett. 93, 125502 (2004).

[37] T. Richeton, J. Weiss, and F. Louchet, Breakdown of avalanche critical behaviour in polycrystalline plasticity, Nat. Mater. 4, 465 (2005).

[38] R Core Team, R: A Language and Environment for Statistical Computing, R Foundation for Statistical Computing, Vienna, Austria, 2016, https://www.R-project.org/.

[39] R. P. Brent, Algorithms for Minimization Without Derivatives (Dover, New York, 2002).

[40] W. H. Press, S. A. Teukolsky, W. T. Vetterling, and B. P. Flannery, Numerical Recipes in FORTRAN, 2nd ed. (Cambridge University, Cambridge, England, 1992). 